\title{
HADES-CCG, A new Tomographic Reconstruction Tool
}

H. E. Martz, Jr., M. B. Aufderheide, III, J. Hall, S. Schach von Wittenau, D. Goodman, C. Logan, J. Jackson, D. Slone

This article was submitted to $27^{\text {th }}$ Annual Review of Progressin Quantitative Nondestructive Evaluation, Ames, lowa, July 16-22, 2000

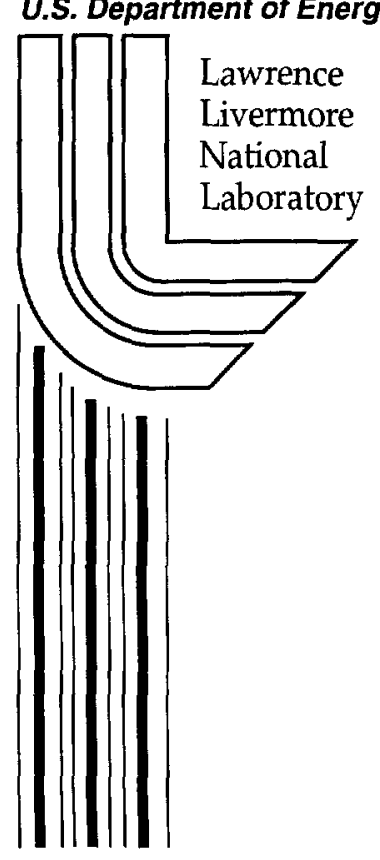




\section{DISCLAIMER}

This document was prepared as an account of work sponsored by an agency of the United States Government. Neither the United States Government nor the University of California nor any of their employees, makes any warranty, express or implied, or assumes any legal liability or responsibility for the accuracy, completeness, or usefulness of any information, apparatus, product, or process disclosed, or represents that its use would not infringe privately owned rights. Reference herein to any specific commercial product, process, or service by trade name, trademark, manufacturer, or otherwise, does not necessarily constitute or imply its endorsement, recommendation, or favoring by the United States Government or the University of California. The views and opinions of authors expressed herein do not necessarily state or reflect those of the United States Government or the University of California, and shall not be used for advertising or product endorsement purposes.

This is a preprint of a paper intended for publication in a journal or proceedings. Since changes may be made before publication, this preprint is made available with the understanding that it will not be cited or reproduced without the permission of the author.

This work was performed under the auspices of the United States Department of Energy by the University of California, Lawrence Livermore National Laboratory under contract No. W-7405-Eng-48.

This report has been reproduced directly from the best available copy.

Available electronically at http://www.doc.gov/bridge

Available for a processing fee to U.S. Department of Energy

And its contractors in paper from

U.S. Department of Energy

Office of Scientific and Technical Information

P.O. Box 62

Oak Ridge, TN 37831-0062

Telephone: (865) 576-8401

Facsimile: (865) 576-5728

E-mail: reports@adonis.osti.gov

Available for the sale to the public from

U.S. Department of Commerce

National Technical Information Service

5285 Port Royal Road

Springfield, VA 22161

Telephone: (800) 553-6847

Facsimile: (703) 605-6900

E-mail: orders@ntis.fedworld.gov

Online ordering: http://www.ntis.gov/ordering.htm

\section{OR}

Lawrence Livermore National Laboratory

Technical Information Department's Digital Library

http://www.llnl.gov/tid/Library.html 


\title{
HADES-CCG, A NEW TOMOGRAPHIC RECONSTRUCTION TOOL
}

\author{
Harry E. Martz, Jr., Maurice B. Aufderheide, III, James Hall, Alexis Schach von Wittenau, \\ Dennis Goodman, Clint Logan, Jessie Jackson and Dale Slone
}

Lawrence Livermore National Laboratory

7000 East Avenue, Livermore, CA 94550 USA

\begin{abstract}
.
We have developed a new tomography code, HADES-CCG. This code uses HADES, a radiographic simulation code, to perform forward- and back-projection and is coupled to a Constrained Conjugate Gradient (CCG) optimizer. An iterative solution to the reconstruction problem is found which is optimal, given the detector noise model, a source model and the appropriate attenuation cross-sections. By explicitly including experimental effects in forward- and back-projection, these effects are not folded back into the object model.
\end{abstract}

\section{INTRODUCTION}

Current tomographic methods used for reconstructing CT images make strong assumptions about the radiographic projection data. These assumptions include the presence of energy-independent photon-attenuation cross sections and the absence of detector or source-spot blur, statistical noise associated with scattering and other artifacts. These assumptions are not strictly correct. Radiation-attenuation cross sections depend strongly on energy and material composition, detector and source-spot blur can be and usually is significant, scattering is present and statistical noise and artifacts are common. Some if not most of these assumptions can be treated in the forward model of a reconstruction algorithm. We seek to improve the accuracy of computed tomography from transmission radiographs by merging a simulation capability (HADES) with a maximumlikelihood constrained-conjugate-gradient (CCG) reconstruction technique, resulting in a physics-based, forward-model image reconstruction code. Current LLNL radiographic modeling contrast sensitivity is $\sim 5-10 \%$ and tomographic reconstructions have an accuracy of $\sim 20 \%$ compared to experimental results. The goal is to improve radiography models so that tomographic image reconstructions are accurate at the $1 \%$ contrast sensitivity level or better. Forward-modeling techniques are being explored elsewhere (e.g., at LANL [1], in the medical imaging community [2, 3], in France [4], Germany/Russia [5] and the Netherlands [6]), but all of these works are limited in scope. This project is unique in its full-physics simulation approach, in its use of CCG to reconstruct $2 \mathrm{D}$ and $3 \mathrm{D}$ meshes, and in the breadth of data to which it will be applied. 


\section{HADES-CCG TOMOGRAPHY CODE}

An improved version of HADES [7] has been coupled with a recently developed CT algorithm known as CCG (Constrained Conjugate Gradient) [8]. The problem of image reconstruction is expressed as a large matrix equation relating a model for the object being reconstructed to its projections (radiographs). Using a constrained-conjugate-gradient search algorithm, a maximum likelihood solution is sought. This search continues until the difference between the input measured radiographs or projections and the simulated or calculated projections is satisfactorily small (see Figure 1).

With respect to Figure 1, our model of the projection measurement is given by

$$
p(\underline{r})=f[o(\underline{r}), g(\underline{r}), b(\underline{r}), n(\underline{r})],
$$

where $\underline{r}$ is the 3D spatial coordinate, $p$ is the measured projection data, $o$ is the object function (including density and material variations), $g$ is the geometry of the CT scanning system, $b$ is the spatial blur and $n$ is the system noise.

HADES and CCG are merged such that each code maintains its individual capability. This approach allows the same version of HADES merged with CCG to be validated against experimental radiographs. HADES does the forward- and back-projection required in the CCG CT image reconstruction algorithm. This more accurate radiographic model will improve object recovery. By identifying and separating each of the experimental effects we expect to achieve very high accuracy in the image reconstructions.

The intent is to recover the object from the projection measurement. For object recovery using tomography, image reconstruction artifacts are an additional error source ("a" in Figure 1). CT image reconstruction artifacts include: narrow streaks caused by baddetector pixels, broad streaks caused by opaque objects, rings caused by an imbalance in detector-to-detector response, cupping caused by a combination of the preferential absorption of the low-energy photons over high-energy photons for a bremsstrahlung $\mathrm{x}$-ray source (sometimes called "beam hardening") and x-ray scattering.

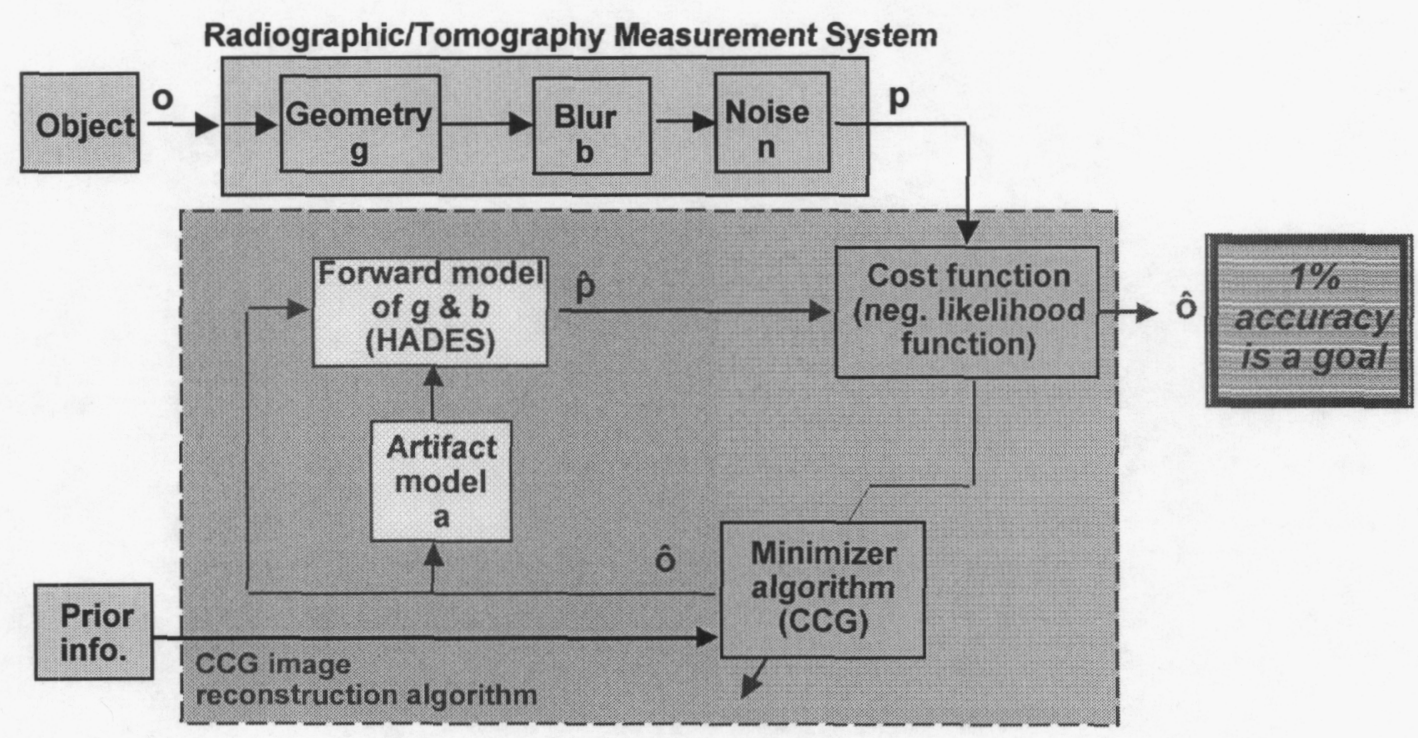

Figure 1. Flow diagram of the process for recovering an object function from radiographic projection data using the HADES-CCG image reconstruction algorithm. The circumflex ( $\left.{ }^{\wedge}\right)$ appearing over some parameters indicates that they are estimated quantities. 
The present implementation of CCG at LLNL ("JCONE-CCG") is a cone-beam projector algorithm that does not incorporate blur or noise models and hence fails to account for these experimental effects. As a result, experimental radiographs (which have blurred and statistical noise fluctuations) processed using JCONE-CCG will yield reconstructions that are blurred and noisy. In addition, JCONE-CCG only reconstructs a 3D Cartesian mesh. In many cases, an object may have an axial symmetry, which would make it desirable to reconstruct into a 2D r-z mesh with a limited number of projections. We are developing a 2D CCG CT code that uses full ray-tracing simulations from HADES as the projector. The physics (e.g., scattering and detector response) required in the HADES code is determined from Monte Carlo simulations [9]. The current version of HADES-CCG reconstructs into a mesh made of one material and assumes a monochromatic source.

\section{TEST OBJECTS}

Radiography and computed tomography test objects are helpful in understanding and validating the simulation codes and have been used to determine the performance of the CCG image reconstruction algorithm with (HADES-CCG) and without (JCONE-CCG) the forward-modeling capabilities. The test objects were designed to meet several criteria: ability to be simulated by Monte Carlo codes and HADES, ease of fabrication, adaptability to different types of problems and the presentation of a challenging imaging situation.

The radiographic test objects used for model validation thus far include a $\mathrm{Cu}$-step wedge, a Ta edge, a Ta slug and an opaque $\mathrm{W}$ rod. An alignment phantom, a D-38 CT object, the British Test Object (BTO) (a set of nested C, W and polyethylene shells) and a W shell have all been used for tomographic verification. Details of these test objects are provided elsewhere [9]. Here we will restrict our discussion to the BTO (Figure 2). The main interest in the BTO is its geometric simplicity, axial symmetry and relatively high areal density $\left(\approx 175 \mathrm{~g} / \mathrm{cm}^{2}\right)$.

\section{EXPERIMENTAL EQUIPMENT}

Both neutrons and $\mathrm{x}$-rays were used to image the BTO. For neutron imaging, an accelerator-driven $\mathrm{D}(\mathrm{d}, \mathrm{n})^{3} \mathrm{He}$ neutron source was used to generate a nearly monoenergetic neutron beam $(10 \mathrm{MeV} \pm 500 \mathrm{keV})$. Neutrons transmitted through the object were imaged by a thick $(4 \mathrm{~cm}) \mathrm{BC}-400$ plastic scintillator viewed by an $\mathrm{LN}_{2}$-cooled $\mathrm{CCD}$ camera fitted with a fast (f/1.0) lens [10]. The active area of the 16-bit CCD array is $2.54 \mathrm{~cm} \mathrm{X} 2.54 \mathrm{~cm}$, with 1024 X 1024 pixels with a $24.8 \mu \mathrm{m}$ pitch. The camera assembly was located off axis in a shielded enclosure and viewed the scintillator via a thin aluminized-Pyrex mirror. The neutron radiograph of the BTO shown in the upper left of Figure 3 was acquired with symmetric 2-m source-to-detector and object-to-detector distances.

For $\mathrm{x}$-ray imaging, a 9-MV electron LINAC (linear accelerator) $\mathrm{x}$-ray source and an amorphous-Si (a-Si) array Flashscan 20 detector made by dpi $\chi$ [11] were employed. The Flashscan 20 is a flat-panel array of light-sensitive photodiodes fabricated using thin film transistors. The active area of the 12-bit a-Si array is $19.5 \mathrm{~cm} \mathrm{X} 24.4 \mathrm{~cm}$, with 1536 X 1920 pixels with a $127 \mu \mathrm{m}$ pitch. This array must be used with a scintillator to convert X-ray radiation energy to visible light. We have selected a commercial mammography "screen", Min-R, as having the best spatial resolution and sensitivity for this application [12]. The dpix imager was equipped with additional 1.2-cm-thick $\mathrm{Pb}$ shielding to protect the electronic components after observing damage in early experiments. The x-ray radiograph of the BTO shown in the bottom left of Figure 3 was acquired with $6-\mathrm{m}$ source-to-detector and $1-\mathrm{m}$ object-to-detector distances. 

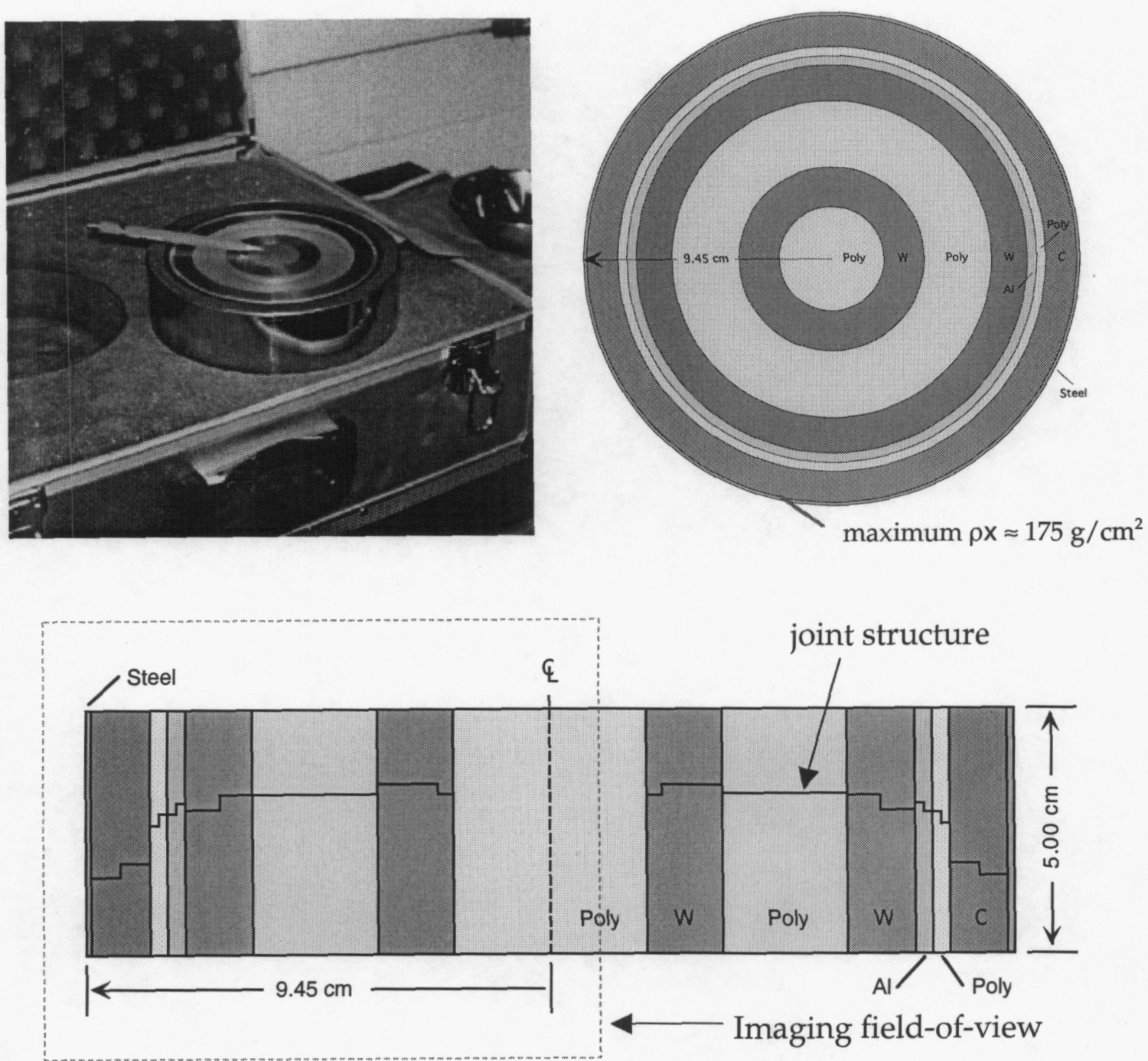

Figure 2. Top: Left: Photograph of the British Test Object (BTO). Right: Top view of the BTO. Bottom: Cross-sectional view of the BTO showing materials and internal joint structure.

\section{RESULTS AND DISCUSSION}

The neutron and $\mathrm{x}$-ray radiographs (Figure 3, left) of the BTO show some of the joint structure and material boundaries. However, it is difficult to use the radiographic projection data to obtain detailed quantitative measurements of the joint thickness, material boundaries, etc. To extract more details from the BTO radiographs, a single radiographic projection was used to obtain a 2D CT cross section of the object (Figure 3, right). These cross sections were obtained using the current HADES-CCG code.

When HADES simulates radiographs, it uses the mass-absorption coefficients from a user-specified data base to determine path lengths [7]. When HADES-CCG produces a reconstruction, this extra knowledge is used to reconstruct into density rather than the linear-attenuation coefficient. This is a useful feature not produced with traditional tomographic reconstruction codes. This feature has been particularly useful in comparing reconstructions from $\mathrm{x}$-ray and neutron radiographs, because HADES uses the appropriate mass-absorption coefficients for each image. 

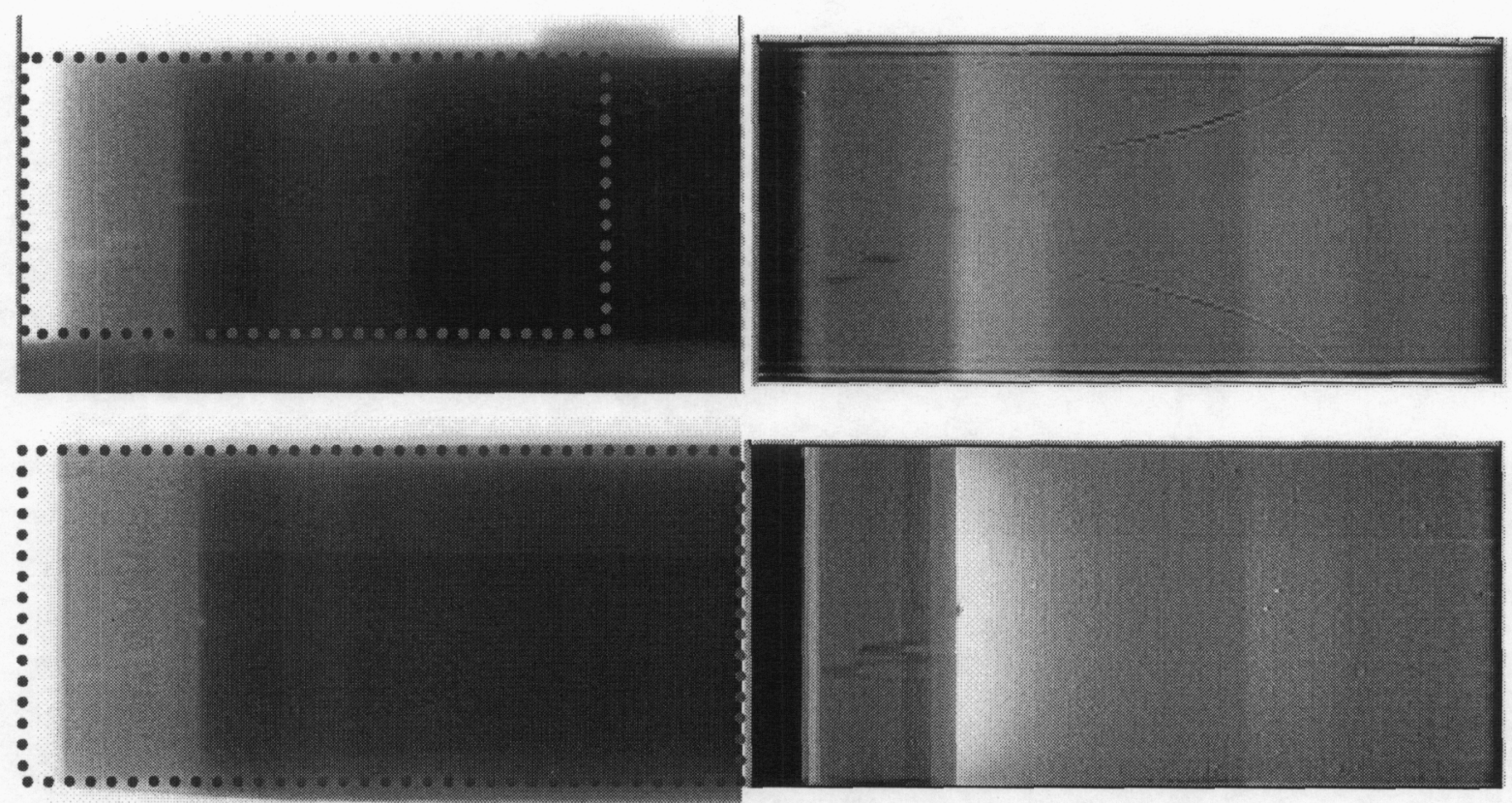

Figure 3. Top: Left: 10-MeV neutron radiograph of the BTO. Right: $2 \mathrm{D}$ CT reconstruction of the BTO using the single radiograph shown to the left. Bottom: Left: 9-MV x-ray radiograph of the BTO. Right: 2D CT reconstruction of the BTO using the single radiograph shown to the left. The dotted box highlights the portion of the radiograph used to obtain the $2 \mathrm{D}$ cross-sectional $\mathrm{CT}$ data in each case. Both CT images were reconstructed into the same voxel size of $0.5 \mathrm{~mm}$ X $0.5 \mathrm{~mm}$.

As mentioned above, the HADES-CCG code reconstructs into a single material mesh assuming a monochromatic source. As shown in Figure 2, the BTO consists of multiple materials. The densities inferred in the reconstruction will only be correct for the single material specified in the reconstruction. However, the error made by this assumption for the other materials is easily corrected by normalizing the mass absorption coefficients for each material not used in the reconstruction. More serious is the neglect of polychromaticity in the reconstruction. The neutrons used varied in energy from 9.5 to $10.5 \mathrm{MeV}$, while the $\mathrm{x}$ ray source had a $9 \mathrm{MV}$ bremsstrahlung spectrum. We intend to include these effects in future refinements of the code.

Comparing the $\mathrm{x}$-ray $\mathrm{CT}$ reconstruction to the neutron $\mathrm{CT}$ reconstruction on the right of Figure 3, it is apparent that spatial resolution is superior in the x-ray image. This is expected and is largely due to source unsharpness. The LINAC source size is nominally 2 $\mathrm{mm}$ which leads to a source unsharpness at the object plane of $0.4 \mathrm{~mm}$. The neutron source size is nominally $3 \mathrm{~mm}$ with a corresponding unsharpness at the object of $1.5 \mathrm{~mm}$. In the $\mathrm{x}$-ray image the shells outside of the outer W shell are more visible and distinct than in the neutron image. The joint structure is clearly visible in the outer shells of the BTO in both images, but is sharper in the $\mathrm{x}$-ray image and has an annular ring shape most likely due to misalignment. The annular ring shape arises from taking a single misaligned radiographic projection and reconstructing into a $2 \mathrm{D}-\mathrm{CT}$ cross section using axial symmetry. The greater utility of $10-\mathrm{MeV}$ neutrons for imaging highly-attenuating objects is shown by the appearance of the inner and outer surfaces of the middle polyethylene shell while the $\mathrm{x}$-ray image shows no evidence of the outer surface.

Several prominent curved and horizontal artifacts appear in the neutron CT reconstruction which do not appear in the x-ray reconstruction (Figure 3, right). There are three principal differences between the two images: (1) the use of neutrons vs. X-rays, (2) different imaging detectors and (3) different imaging cone angles. We do not think that the artifacts are due to the use of neutrons vs. x-rays or the different imaging detectors used. 
Some preliminary studies have shown that the curved artifacts in the neutron image may be due to large cone angles and interpolation scheme used to reconstruct the projection data. The neutron data have a cone angle of $1.4^{\circ}$ while the $\mathrm{x}$-ray data have an angle of $0.57^{\circ}$. Further work is required to determine if the larger cone angle is the cause of the curved artifacts. The horizontal artifacts are visible in both images but are more noticeable in the neutron reconstruction. They are most likely due to cropping the radiographic data before reconstruction. Because we were interested in reconstructing the volume of the object and not its upper and lower edges, we deliberately cropped the parts of the radiograph showing the upper and lower edges of the object. This introduces an inconsistency of the data that manifests itself as the horizontal line artifacts. This inconsistency becomes worse as cone angle increases, hence the artifact is more noticeable in the neutron reconstruction.

\section{FUTURE WORK}

Our current implementation of HADES-CCG has shown interesting potential, but more work is needed to make a fully viable reconstruction code. One area that requires more work is the treatment of multiple materials in a reconstruction. The general problem of inferring multiple materials from radiographic projections is too ill-posed to allow a unique reconstruction. We will use prior knowledge in future experiments to constrain the reconstruction.

Another area that requires more work is the treatment of polychromatic sources. Because most imaging detectors measure absorbed energy rather than the energy spectrum, we again have an extremely ill-posed problem to solve. Here we also plan to use prior knowledge about the source energy spectrum and detector spectral response in order to obtain a unique reconstruction.

All of the discussions in this paper have been for $2 \mathrm{D}$ reconstructions. We started with $2 \mathrm{D}$ reconstructions because it allows us to concentrate on the physics of the problem while reducing the size of the data set. We will expand the HADES-CCG code to include 3D reconstructions in the future. This will broaden the applicability of the code to nonsymmetrical objects. We anticipate that the above-mentioned work on multiple materials and polychromaticity will carry over to $3 \mathrm{D}$ reconstructions.

All of our current work has used the fairly standard practice of taking logarithms of images, so that the tomography problem can be expressed as a linear matrix equation; however, the true transmission radiography process is non-linear because Beer's law actually yields an integral (over source energy) of exponential attenuation terms. Such a system does not yield the simple linear matrix equation, which is usually solved in tomography. Complex cross terms are produced in the process of forming derivatives and gradients. This approach is worth investigating because it is the only way to accurately treat the detector noise model in the simulation. The traditional linearized approach (i.e. taking logarithms of data corrupted with noise) makes detector noise models very difficult to treat.

\section{SUMMARY}

We have successfully coupled the ray-tracing code HADES with the constrained conjugate gradient optimizer CT image reconstruction code CCG. HADES and CCG have been merged such that each code maintains its individual capability. This approach allows the same version of HADES merged with CCG to be validated against experimental radiographs. HADES does the forward- and back-projection required in the CCG CT image reconstruction algorithm. The physics (e.g., scattering and detector response) required in HADES is determined from Monte Carlo simulations. The current version of HADES- 
CCG reconstructs into a mesh made of one material and assumes a monochromatic source. We have just begun validating the HADES-CCG reconstruction code on both neutron and $x$-ray projection data. Preliminary 2D CT images have been used to test this code on neutron and x-ray experimental single-view projection data for a cylindrical test object. The results are encouraging; however, a lot more work is required. For example, we need to extend the code to $3 \mathrm{D}$ and multiple materials using polychromatic sources.

\section{ACKNOWLEDGMENTS}

The authors would like to thank Derrill Rikard, Earl Updike, Kenn Morales, Alex Guerrero and Dan Schneberk for their help in acquiring and processing the digital x-ray radiographic data. This work was performed under the auspices of the U.S. Department of Energy by The University of California Lawrence Livermore National Laboratory under contract No. W-7405-ENG-48.

\section{REFERENCES}

1. Hanson, K. M., Cunningham, G. S., and Saquib, S. S., "Inversion based on computational simulations" to be published in Maximum Entropy and Bayesian Methods, edited by G. Erickson, et al., Kluwer Academic, Dordrecht, (1998).

2. Kaplan, M. S,. et al., "Scatter and Attenuation Correction for " In Based on Energy Spectrum Fitting," Med. Phys., Vol. 23, (1996) pp. 200-214.

3. Cunningham, G. S., and Hanson, K. M., "Three-dimensional reconstructions from low-count SPECT data using deformable models," Optics Express 2, pp. 227-236, (1998).

4. Lagrange, J. M., Dauty, I., and Azencott, R., "Tomographic reconstruction of axisymmetrical objects from one view by model approximation," 1997 IEEE Image Processing Conference, p. 492 (1997).

5. Vengrinovich, V. L., Denkevich, Y. B., Tillack, G. R. and Jacobsen, C., in Review of Progress in $Q N D E$, edited by D. O. Thompson and D. E. Chimenti, Plenum, New York, 1999, Vol. 18, p. 935.

6. Leliveld, C. J., Maas, J. G., Bom, V. R., and van Eijk, C. W. E., "The effects of detected scattered radiation and beam hardening in $\mathrm{x}$-ray computerized tomographic imaging of industrial objects," in Proceedings of the International Symposium on Computerized Tomography for Industrial Applications, June 8-10, 1994, Berlin, Germany.

7. Aufderheide, M. B., Slone, D., and Schach von Wittenau, A., "HADES A New Radiographic Simulation Code," in these proceedings.

8. Goodman, D. M., Johansson, E. M., and Lawrence, T. W., "On Applying the Conjugate Gradient Algorithm to Image Processing Problems," Chapter 11 in Multivariate Analysis: Future Directions, edited by C. R. Rao, Elsevier Science Publishers, North Holland, 1993, pp. 209-232.

9. Martz, Jr., H. E., Aufderheide, III, M. B., Goodman, D., Schach von Wittenau, A., Logan, C., Hall, J. and Jackson, J. A., "Quantitative Tomography Simulations and Reconstruction Algorithms," in Engineering Research, Development and Technology FY 99, Lawrence Livermore National Laboratory, Livermore, CA, UCRL 53868-99, 2000.

10. Hall, J., Dietrich, F., Logan, C., and Schmid, G., "Development of high-energy neutron imaging for use in NDE applications," SPIE 3769, pp. 31-42 (1999). 
11. Weisfield, R. L., Hartney, M. A., Street, R. A., and Apte, R. B., "New AmorphousSilicon Image Sensor for X-Ray Diagnostic Medical Imaging Applications," SPIE Medical Imaging, Physics of Medical Imaging, Vol. 3336, pp. 444-452 (1998).

12. Dolan, K., Logan, C.. Haskins, J., Rikard, D., and Schneberk, D., "Evaluation of an Amorphous Silicon Array for Industrial X-ray Imaging"in Engineering Research, Development and Technology FY 99, Lawrence Livermore National Laboratory, Livermore, CA, UCRL 53868-99, 2000. 